\title{
Substituent Effects in the Hydrosilylation of Coordinated Dinitrogen in a Ditantalum Complex: Cleavage and Functionalization of $\mathbf{N}_{2}$
}

\author{
Bruce A. MacKay, Rui F. Munha, and Michael D. Fryzuk*
}

\author{
Department of Chemistry \\ The University of British Columbia \\ 2036 Main Mall \\ Vancouver, BC Canada \\ V6T $1 \mathrm{Z1}$
}

E-mail: fryzuk@chem.ubc.ca

\section{Synthesis of $[\mathrm{NPN}] \mathrm{Ta}(\mathrm{H})(\mu-\mathrm{H})_{2}\left(\mu-\eta^{1}: \eta^{2}-\mathrm{N}_{2}-\mathrm{SiH}_{2}{ }^{\mathrm{n}} \mathrm{Bu}\right) \mathrm{Ta}[\mathrm{NPN}], 5$}

To a stirred solution of $758 \mathrm{mg}(0.601 \mathrm{mmol}) 1 \mathrm{in} 30 \mathrm{~mL}$ toluene was added $53.2 \mathrm{mg}(0.603$ mmol, $\sim 1$ equiv) of butylsilane in roughly $3 \mathrm{~mL}$ toluene in a glove box. The flask was stored for $24 \mathrm{~h}$ in a $-60^{\circ} \mathrm{C}$ freezer, after which the dark brown solution had turned red-orange. Solvent was removed under vacuum, leaving a pinkish residue which was triturated under pentane. The resulting precipitate was recovered on a glass frit, yielding $729 \mathrm{~g}(0.593 \mathrm{mmol}$, 98.6\% yield) 5. ${ }^{1} \mathrm{H} \mathrm{NMR}\left(\mathrm{C}_{7} \mathrm{D}_{8},-60{ }^{\circ} \mathrm{C}, 400 \mathrm{MHz}\right): \delta-0.17,-0.09,0.01,0.02,0.04,0.40$ (s, $3 \mathrm{H}$ each) $0.03\left(\mathrm{~s}, 6 \mathrm{H}\right.$, total $\left.24 \mathrm{SiCH}_{3}\right), 0.72,1.05,0.46,1.23,0.78,1.29,1.20$, and 1.81 (AMX, 1H each, $\mathrm{SiCH}_{2} \mathrm{P}$ ), 5.05 and $3.97\left(\mathrm{~d},{ }^{2} \mathrm{~J}_{\mathrm{HH}} 11.7 \mathrm{~Hz}, 1 \mathrm{H}\right.$ each, $\mathrm{NSi} H_{2} \mathrm{Bu}$ ), -0.25 (br, $2 \mathrm{H}, \mathrm{SiCH}_{2} \mathrm{CH}_{2-}$ ), 0.74 (br m, $2 \mathrm{H},-\mathrm{CH}_{2} \mathrm{CH}_{2} \mathrm{CH}_{2}-$ ), 1.05 (br, $2 \mathrm{H},-\mathrm{CH}_{2} \mathrm{CH}_{2} \mathrm{CH}_{3}$ ), 0.81 (d, 3H, $\left.\mathrm{CH}_{2} \mathrm{CH}_{3}\right), 7.13,7.01,7.79,7.20,7.33,7.42,7.07,7.13,7.225,6.93,7.32,7.50,7.24,7.34$, 7.19, and 7.24 (overlapping m, PPh- $H$ and NPh- $H$ ) 8.342 and 7.77 (m, 2H each, PPh-o-H), 11.21 and 11.23 (ddd, $1 \mathrm{H}$ each, $\left.\mathrm{J}_{\mathrm{HbHb}}=3.8 \mathrm{~Hz},{ }^{2} \mathrm{~J}_{\mathrm{HbHt}}=5.52 \mathrm{~Hz},{ }^{2} \mathrm{~J}_{\mathrm{HP}}=11.7 \mathrm{~Hz}, \mathrm{TaHTa}\right)$ $14.27\left(\mathrm{dd},{ }^{2} J_{\mathrm{HP}}=17.99 \mathrm{~Hz}, J_{\mathrm{HH}}=5.52 \mathrm{~Hz}, 1 \mathrm{H}, \mathrm{Ta}-\mathrm{H}_{\mathrm{t}}\right) .{ }^{13} \mathrm{C} \mathrm{NMR}\left(\mathrm{C}_{7} \mathrm{D}_{8},-60{ }^{\circ} \mathrm{C}, 100.61\right.$ MHz): 0.29, 0.41, 0.49, 1.11, 2.21, 2.38, 3.27, 3.93 (s or d, $\mathrm{SiCH}_{3}$ ), 14.98, 23.61, 26.20, 32.61 (br s, $\left.\mathrm{SiCH}_{2} \mathrm{P}\right), 1.81 \quad\left(\mathrm{~s}, \mathrm{SiCH}_{2} \mathrm{CH}_{2}-\right), 34.81$ (s, $-\mathrm{CH}_{2} \mathrm{CH}_{2} \mathrm{CH}_{2-}$ ), 18.54 (s, $\left.\mathrm{CH}_{2} \mathrm{CH}_{2} \mathrm{CH}_{3}\right), 14.09$ (s, $\left.\mathrm{CH}_{2} \mathrm{CH}_{3}\right), 127.47,121.82,128.52,122.19,129.46,134.03,121.33$, and $134.93\left(\mathrm{P}\left(\mathrm{C}_{6} \mathrm{H}_{5}\right)\right.$ and $\mathrm{N}\left(\mathrm{C}_{6} \mathrm{H}_{5}\right) .{ }^{31} \mathrm{P}\left\{{ }^{1} \mathrm{H}\right\} \mathrm{NMR}\left(\mathrm{C}_{7} \mathrm{D}_{8},-60{ }^{\circ} \mathrm{C}, 161.97 \mathrm{MHz}\right): \delta 23.32(\mathrm{~d}$, $\left.J_{\mathrm{PP}}=17.83 \mathrm{~Hz}\right), 8.91\left(\mathrm{~d}, J_{\mathrm{PP}}=17.83 \mathrm{~Hz}\right) .{ }^{29} \mathrm{Si} \mathrm{NMR}\left(\mathrm{C}_{7} \mathrm{D}_{8},-60{ }^{\circ} \mathrm{C}, 79.5 \mathrm{MHz}\right) \delta-14.96(\mathrm{~s}$, $\left.\mathrm{NSiH} \mathrm{H}_{2} \mathrm{Bu}\right) 11.17\left(\mathrm{~d},{ }^{2} \mathrm{~J}_{\mathrm{PSi}}=11.3 \mathrm{~Hz}\right), 8.98\left(\mathrm{~d},{ }^{2} \mathrm{~J}_{\mathrm{PSi}}=9.6 \mathrm{~Hz}\right) 8.79\left(\mathrm{~d},{ }^{2} \mathrm{~J}_{\mathrm{PSi}}=8.7 \mathrm{~Hz}\right), 8.49(\mathrm{~d}$, 
${ }^{2} \mathrm{~J}_{\mathrm{PSi}}=14.3 \mathrm{~Hz}$ ). UV-Vis: $\lambda_{\max }=500 \mathrm{~nm}, \varepsilon=577 \mathrm{M}^{-1} \mathrm{~cm}^{-1}$. Anal. Calc'd for $\mathrm{C}_{52} \mathrm{H}_{76} \mathrm{~N}_{6} \mathrm{P}_{2} \mathrm{Si}_{5} \mathrm{Ta}_{2}$ : C 46.28; H 5.68; N 6.23. Found: C 45.98; H 5.45; N 6.48.

\section{Synthesis of ${ }^{15} \mathrm{~N}_{2}-5$.}

A solution of ${ }^{15} N_{2}-\mathbf{1}$ was treated in a manner similar to the preparation of $5 .{ }^{15} \mathrm{~N} N M R\left(\mathrm{C}_{7} \mathrm{D}_{8}\right.$, $\left.-60{ }^{\circ} \mathrm{C}, 40 \mathrm{MHz}\right) \delta-163.5\left(\mathrm{~d},{ }^{1} \mathrm{~J}_{\mathrm{NN}}=16.6 \mathrm{~Hz}\right)-52.5\left(\mathrm{dd},{ }^{2} \mathrm{~J}_{\mathrm{PN}}=26.4 \mathrm{~Hz},{ }^{1} \mathrm{~J}_{\mathrm{NN}}=16.6 \mathrm{~Hz}\right)$.

Additional coupling of $26.4 \mathrm{~Hz}$ was observed in the $\delta 23.32 \mathrm{ppm}$ resonance in the ${ }^{31} \mathrm{P}\left\{{ }^{1} \mathrm{H}\right\}$ spectrum, as was an additional coupling of $5.2 \mathrm{~Hz}$ in the $\delta-14.96 \mathrm{ppm}$ resonance of the ${ }^{29} \mathrm{Si}\left\{{ }^{1} \mathrm{H}\right\}$ spectrum.

\section{Synthesis of $[\mathrm{NPN}] \mathrm{Ta}(\mathrm{H})(\mu-\mathrm{N})\left(\mu-\mathrm{N}-\mathrm{SiH}_{2}{ }^{\mathrm{B}} \mathrm{Bu}\right) \mathrm{Ta}[\mathrm{NPN}], 6$.}

A red/orange $15 \mathrm{~mL}$ toluene solution of $7(231 \mathrm{mg}, 0.188 \mathrm{mmol})$ was left at $15^{\circ} \mathrm{C}$ in a glove box for $36 \mathrm{~h}$, after which the solvent was removed under vacuum, leaving a yellow-brown residue which was triturated under hexanes. Fine yellow-white needles of $\mathbf{6}$ were recovered on a glass frit (212 mg, $0.173 \mathrm{mmol}, 92 \%$ yield). ${ }^{1} \mathrm{H}$ NMR $\left(\mathrm{C}_{6} \mathrm{D}_{6}, 30^{\circ} \mathrm{C}, 400 \mathrm{MHz}\right): \delta-0.12$, $0.06,0.17$ and 0.38 (s, $6 \mathrm{H}$ each, $24 \mathrm{H}$ total, $\left.\mathrm{SiCH}_{3}\right), 1.00,1.26,1.22$, and 1.43 (d, $2 \mathrm{H}$ each, $\mathrm{SiCH}_{2} \mathrm{P}$ ), 4.38 (b, $2 \mathrm{H}, \mathrm{NSi} \mathrm{H}_{2} \mathrm{Bu}$ ), -0.18 (br, $2 \mathrm{H}, \mathrm{SiCH}_{2} \mathrm{CH}_{2}$ ), 0.78 (br m, $2 \mathrm{H},-\mathrm{CH}_{2} \mathrm{CH}_{2} \mathrm{CH}_{2}-$ ), 1.11 (br, $2 \mathrm{H},-\mathrm{CH}_{2} \mathrm{CH}_{2} \mathrm{CH}_{3}$ ), 0.86 (d, $3 \mathrm{H}, \mathrm{CH}_{2} \mathrm{CH}_{3}$ ), 7.05, 7.19, 7.38, 7.47, 6.94, 6.98, 7.12, 7.26, 7.36, and 7.53 (overlapping $\mathrm{m}, \mathrm{PPh}-H$ and $\mathrm{NPh}-H) 7.85$ and 7.30 (m, 2H each, PPh-oH), $17.27\left(\mathrm{~d},{ }^{2} J_{\mathrm{HP}}=41.14 \mathrm{~Hz}, 1 \mathrm{H}, \mathrm{Ta}-\mathrm{H}_{\mathrm{t}}\right) .{ }^{13} \mathrm{C}$ NMR $\left(\mathrm{C}_{6} \mathrm{D}_{6}, 30{ }^{\circ} \mathrm{C}, 100.61 \mathrm{MHz}\right): \delta 0.37$, 0.98, 1.29, 1.41, (s, $\left.\mathrm{SiCH}_{3}\right), 22.12$ and 31.45 (br s, $\left.\mathrm{SiCH}_{2} \mathrm{P}\right), 2.88$ (s, $\left.\mathrm{SiCH}_{2} \mathrm{CH}_{2}-\right), 25.47$ (s, $\mathrm{CH}_{2} \mathrm{CH}_{2} \mathrm{CH}_{2}$ ), 14.39 (s, - $\mathrm{CH}_{2} \mathrm{CH}_{2} \mathrm{CH}_{3}$ ), 22.44 (s, $\left.\mathrm{CH}_{2} \mathrm{CH}_{3}\right), 116.66,121.58,122.41,127.33$, 127.90, 1288.36, 129.57, 133.12 (broad overlapping resonances, $\mathrm{P}\left(C_{6} \mathrm{H}_{5}\right)$ and $\mathrm{N}\left(C_{6} \mathrm{H}_{5}\right)$ ). ${ }^{31} \mathrm{P}\left\{{ }^{1} \mathrm{H}\right\}$ NMR $\left(\mathrm{C}_{6} \mathrm{D}_{6}, 30^{\circ} \mathrm{C}, 161.97 \mathrm{MHz}\right): \delta-5.73$ (b, FWHM $\left.28 \mathrm{~Hz}\right),-12.26$ (b, FWHM 28 $\mathrm{Hz}) .{ }^{29} \mathrm{Si} \mathrm{NMR}\left(\mathrm{C}_{6} \mathrm{D}_{6}, 30{ }^{\circ} \mathrm{C}, 79.5 \mathrm{MHz}\right) \delta-31.47$ (s, NSiH $\left.\mathrm{H}_{2} \mathrm{Bu}\right) 11.29\left(\mathrm{~d},{ }^{2} \mathrm{~J}_{\mathrm{PSi}}=11.3 \mathrm{~Hz}\right)$, $11.47\left(\mathrm{~d},{ }^{2} \mathrm{~J}_{\mathrm{PSi}}=12.0 \mathrm{~Hz}\right)$. Anal. Calc'd for $\mathrm{C}_{52} \mathrm{H}_{74} \mathrm{~N}_{6} \mathrm{P}_{2} \mathrm{Si}_{5} \mathrm{Ta}_{2}$ : C 46.35; H 5.54; $\mathrm{N} 6.24$. Found: C 46.04; H 5.24; N 5.94.

\section{Synthesis of ${ }^{15} \mathrm{~N}_{2}-6$}

A $\mathrm{C}_{6} \mathrm{D}_{6}$ solution of roughly $40 \mathrm{mg}(0.03 \mathrm{mmol}){ }^{15} \mathrm{~N}_{2}-5$ in a Wilmad NMR tube was allowed to decompose overnight at $15^{\circ} \mathrm{C}$ in a glove box. ${ }^{15} \mathrm{~N}$ NMR $\left(\mathrm{C}_{6} \mathrm{D}_{6}, 30{ }^{\circ} \mathrm{C}, 40 \mathrm{MHz}\right) \delta 284.4$ (b) - 
$44.8\left(\mathrm{~d},{ }^{2} \mathrm{~J}_{\mathrm{PN}}=18.7 \mathrm{~Hz}\right.$,). Additional coupling of $18.7 \mathrm{~Hz}$ was observed in the $\delta-12.26 \mathrm{ppm}$ resonance in the ${ }^{31} \mathrm{P}\left\{{ }^{1} \mathrm{H}\right\}$ spectrum, as was an additional coupling of $4.4 \mathrm{~Hz}$ in the $\delta-31.47$ ppm resonance of the ${ }^{29} \mathrm{Si}\left\{{ }^{1} \mathrm{H}\right\}$ spectrum.

\section{Synthesis of $([\mathrm{NPN}] \mathrm{Ta})_{2}\left(\mu-\mathrm{N}-\mathrm{SiH}_{2} \mathrm{Bu}\right)_{2}, 4$}

To a stirred solution of $488 \mathrm{mg}(0.387 \mathrm{mmol}) 1 \mathrm{in} 10 \mathrm{~mL}$ toluene was added $75.4 \mathrm{mg}(0.855$ mmol, 2.2 equiv) of butylsilane in roughly $2 \mathrm{~mL}$ toluene in a glove box. The dark brown solution turned dark red over the course of $36 \mathrm{~h}$ and solvent was removed under vacuum, leaving a red/purple residue that was triturated under hexanes. The resulting precipitate was recovered on a glass frit and dissolved in roughly $10 \mathrm{~mL} \mathrm{1:1} \mathrm{benzene/hexamethyldisiloxane,}$ and $323 \mathrm{mg}(0.245 \mathrm{mmol}, 63 \%$ yield $)$ of pure crystalline 4 was recovered after slow evaporation. Alternately, $21 \mathrm{mg}(0.23 \mathrm{mmol})$ butylsilane was added dropwise to a stirred 20 $\mathrm{mL}$ toluene solution of $308 \mathrm{mg}(0.229 \mathrm{mmol}, 1$ equiv) 6 . The solution was stirred and turned deep red over four days. Solvent was evaporated and the dark solids were triturated under pentane, giving $271 \mathrm{mg} 4$ (0.201 mmol, 88\% yield). ${ }^{1} \mathrm{H}\left\{{ }^{31} \mathrm{P}\right\} \mathrm{NMR}\left(\mathrm{C}_{6} \mathrm{D}_{6}, 30{ }^{\circ} \mathrm{C}, 400 \mathrm{MHz}\right)$ : $\delta-0.01,0.40,\left(\mathrm{~s}, 12 \mathrm{H}\right.$ each, $\left.\mathrm{SiCH}_{3}\right), 1.60$ and $1.71 \mathrm{ppm}\left(\mathrm{d}, 4 \mathrm{H}\right.$ each, $\left.{ }^{2} \mathrm{~J}_{\mathrm{HH}}=14.1 \mathrm{~Hz}, \mathrm{SiCH}_{2} \mathrm{P}\right)$, -0.31 (br, $2 \mathrm{H}, \mathrm{SiCH}_{2} \mathrm{CH}_{2}-$ ), 0.66 (br m, 2H, $-\mathrm{CH}_{2} \mathrm{CH}_{2} \mathrm{CH}_{2}$ ), 1.10 (br, $2 \mathrm{H},-\mathrm{CH}_{2} \mathrm{CH}_{2} \mathrm{CH}_{3}$ ), 0.856 (d, 3H, $\left.\mathrm{CH}_{2} \mathrm{CH}_{3}\right), 4.91$ (s, 2H, $\left.\mathrm{Si} H_{2}\right) 6.941$ (d, 4H, $\left.p-H \mathrm{PhN}\right), 7.11$ (dd, 8H, $\left.m-H \mathrm{PhN}\right)$, $6.52(\mathrm{~d}, 8 \mathrm{H}, m-H \mathrm{PhN}), 7.12(\mathrm{~d}, 2 \mathrm{H}, p-H \mathrm{PhP}), 7.20(\mathrm{dd}, 4 \mathrm{H}, m-H \mathrm{PhP})$ and $7.65(\mathrm{~d}, 2 \mathrm{H}, o-$ HPhP $).{ }^{31} \mathrm{P}\left\{{ }^{1} \mathrm{H}\right\}$ NMR $\left(\mathrm{C}_{6} \mathrm{D}_{6}, 30{ }^{\circ} \mathrm{C}, 161.97 \mathrm{MHz}\right): \delta 0.08 \mathrm{ppm}(\mathrm{s}) .{ }^{29} \mathrm{Si} \mathrm{NMR}\left(\mathrm{C}_{6} \mathrm{D}_{6}, 30{ }^{\circ} \mathrm{C}\right.$, $79.5 \mathrm{MHz}) \delta 8.70,\left(\mathrm{~d},{ }^{2} \mathrm{~J}_{\mathrm{PSi}}=7.0 \mathrm{~Hz}\right),-1.74\left(\mathrm{dd},{ }^{2} \mathrm{~J}_{\mathrm{PSi}}=4.1 \mathrm{~Hz}, \mathrm{dd},{ }^{2} \mathrm{~J}_{\mathrm{PSi}}=3.9 \mathrm{~Hz}\right)$ Anal. Calc'd for $\mathrm{C}_{56} \mathrm{H}_{84} \mathrm{~N}_{6} \mathrm{P}_{2} \mathrm{Si}_{6} \mathrm{Ta}_{2}$ : C 46.91; H 5.91; N 5.86. Found: C 47.10; H 5.95; N 6.06. Mass Spec (EI/MS) M/z $1383.26(100 \%)$.

\section{Synthesis of ${ }^{15} \mathrm{~N}_{2}-4$.}

A solution of ${ }^{15} N_{2}-\mathbf{1}$ was treated in a manner similar to the preparation of $4 .{ }^{15} \mathrm{~N} N M R\left(\mathrm{C}_{6} \mathrm{D}_{6}\right.$, $\left.30{ }^{\circ} \mathrm{C}, 40 \mathrm{MHz}\right) \delta-23.4\left(\mathrm{dd}, \mathrm{J}_{\mathrm{PN}}=5.1 \mathrm{~Hz}, 11.4 \mathrm{~Hz}\right)$. Additional couplings of 5.1 and $11.4 \mathrm{~Hz}$ were observed in the ${ }^{31} \mathrm{P}\left\{{ }^{1} \mathrm{H}\right\}$ spectrum. The $\delta-1.74 \mathrm{ppm}$ resonance of the ${ }^{29} \mathrm{Si}\left\{{ }^{1} \mathrm{H}\right\}$ spectrum was rendered broad by an unresolvable coupling to ${ }^{15} \mathrm{~N}$.

\section{Synthesis of $\left[\mathrm{NP}\left(\mathrm{C}_{6} \mathrm{H}_{4}\right) \mathrm{N}\right] \mathrm{Ta}\left(\mu-\mathrm{N}-\mathrm{SiH}_{2}{ }^{\mathrm{t}} \mathrm{Bu}\right)\left({ }^{\mathrm{t}} \mathrm{BuSi}(\mathrm{H}) \mathrm{N}=\right) \mathrm{Ta}[\mathrm{NPN}]$.}


$33.7 \mathrm{mg}(0.382 \mathrm{mmol}) t$-butylsilane was added to a stirred $10 \mathrm{~mL}$ toluene solution of $241 \mathrm{mg}$ (0.191 mmol, 0.5 equiv) 1. The stirred solution turned red over 6 hours, and brown thereafter. Solvent was evaporated and the dark solids were triturated under hexanes. After standing overnight, X-ray quality crystals of the product (101 mg, $0.706 \mathrm{mmol}, 37 \%$ yield) were recovered on a frit. In the assignment of the ${ }^{1} \mathrm{H}$ NMR spectrum, the protons on the metalated phenyl ring are labeled $\alpha, \beta, \delta$ and $\gamma$, with the proton nearest the metalated carbon labeled $\alpha .{ }^{1} \mathrm{H}\left\{{ }^{31} \mathrm{P}\right\} \mathrm{NMR}\left(\mathrm{C}_{4} \mathrm{D}_{8} \mathrm{O}, 30^{\circ} \mathrm{C}, 500 \mathrm{MHz}\right): \delta-0.70,-0.67,-0.10,0.01,0.02,0.04$, 0.21, 0.32 (s, 3H each, $\mathrm{SiCH}_{3}$ ), 0.46, 0.86 (s, 9H each, $\left(\mathrm{CH}_{3}\right)_{3} \mathrm{CSi}$ ), 0.33, 0.66, 0.73, 1.12, 1.14, 1.38, 1.45, $1.80\left(\mathrm{~d}, 1 \mathrm{H}\right.$ each, $\left.-\mathrm{CH}_{2} \mathrm{P}\right), 3.99,4.27\left(\mathrm{~d}, 2 \mathrm{~J}_{\mathrm{HH}}=12.7 \mathrm{~Hz},{ }^{\mathrm{t}} \mathrm{BuSiH}_{2}\right), 4.93(\mathrm{~s}$, $\left.1 \mathrm{H}, \mathrm{N}^{\mathrm{t} B u S i}(\mathrm{H}) \mathrm{N}\right), 7.46\left(\mathrm{dd}, 1 \mathrm{H}, \mathrm{H}_{\alpha}\right), 7.80\left(\mathrm{~d}, 1 \mathrm{H}, \mathrm{H}_{\beta}\right), 8.04\left(\mathrm{~d}, 1 \mathrm{H}, \mathrm{H}_{\gamma}\right), 5.89\left(\mathrm{~d}, 1 \mathrm{H}, \mathrm{H}_{\delta}\right)$,

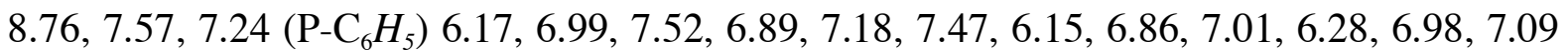
(d, t, 20H total, $\left.\mathrm{N}-\mathrm{C}_{6} H_{5}\right) .{ }^{31} \mathrm{P}\left\{{ }^{1} \mathrm{H}\right\} \mathrm{NMR}\left(\mathrm{C}_{4} \mathrm{D}_{8} \mathrm{O}, 30{ }^{\circ} \mathrm{C}, 202 \mathrm{MHz}\right): \delta-31.4 \mathrm{ppm}(\mathrm{s}) 84.3 \mathrm{ppm}$ (s). Elemental analysis was not obtained.

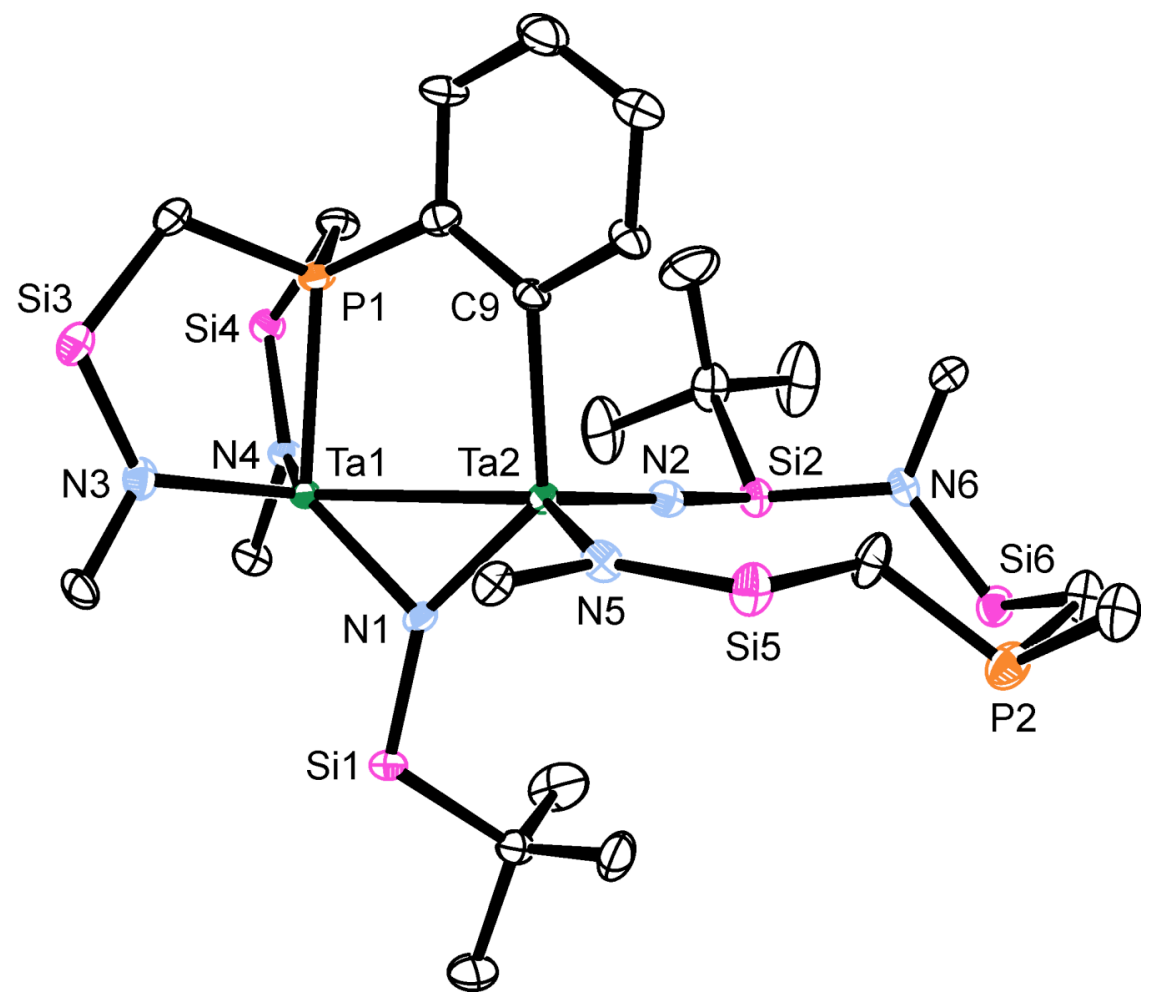

Figure S-1. ORTEP drawing of the solid-state molecular structure of $\left.\left[\mathrm{NP}\left(\mathrm{C}_{6} \mathrm{H}_{4}\right) \mathrm{N}\right] \mathrm{Ta}\left(\mu-\mathrm{N}-\mathrm{SiH}_{2}{ }^{\mathrm{t}} \mathrm{Bu}\right){ }^{\mathrm{t}} \mathrm{BuSi}(\mathrm{H}) \mathrm{N}=\right) \mathrm{Ta}$ [NPN] $]$ (ellipsoids at $50 \%$ probability). Silyl methyls and [NPN] ligand phenyl ring carbons other than ipso omitted for clarity. 
Table S-1. Selected bond distances $(\AA)$, bond angles $\left({ }^{\circ}\right)$, and dihedral angles $\left(^{\circ}\right)$ for $[\mathrm{NP}(\mathrm{Ph}) \mathrm{N}] \mathrm{Ta}\left(\mu-\mathrm{N}-\mathrm{SiH}_{2}{ }^{\mathrm{t}} \mathrm{Bu}\right)\left({ }^{\mathrm{t}} \mathrm{BuSi}(\mathrm{H}) \mathrm{N}=\right) \mathrm{Ta}[\mathrm{NPN}]$.

\begin{tabular}{lll||lll}
\hline Atom & Atom & Distance $(\AA)$ & Atom & Atom & Distance $(\AA)$ \\
\hline Ta1 & Ta2 & $2.9517(10)$ & Ta1 & N1 & $1.977(4)$ \\
Ta2 & N1 & $2.048(5)$ & N1 & Si1 & $1.729(5)$ \\
Ta2 & N2 & $1.807(5)$ & N2 & Si2 & $1.759(5)$ \\
Si2 & N6 & $1.718(5)$ & Ta1 & P1 & $2.4817(17)$ \\
Ta2 & C9 & $2.274(5)$ & & & \\
\hline
\end{tabular}

\begin{tabular}{llll||llll}
\hline Atom & Atom & Atom & Angle $\left(^{\circ}\right)$ & Atom & Atom & Atom & Angle $\left(^{\circ}\right)$ \\
\hline Ta1 & N1 & Ta2 & $94.31(19)$ & Ta1 & N1 & Si & $120.5(3)$ \\
N3 & Ta1 & N4 & $106.49(19)$ & N2 & Ta2 & N5 & $108.3(2)$ \\
\hline
\end{tabular}

\begin{tabular}{lllll}
\hline Atom & Atom & Atom & Atom & Dihedral Angle $\left(^{\circ}\right)$ \\
\hline P1 & Ta1 & Ta2 & N1 & $179.21(19)$ \\
N1 & Ta1 & Ta2 & C9 & $172.7(2)$ \\
\hline
\end{tabular}

\section{General Considerations for X-ray Crystal Structure Determinations}

In all cases, suitable crystals were selected and mounted on a glass fiber using Paratone- $\mathrm{N}$ oil or an acceptable substitute and freezing to $-100{ }^{\circ} \mathrm{C}$. All measurements were made on a Rigaku/ADSC CCD area detector with graphite monochromated Mo-K $\alpha$ radiation. Crystallographic data and some details of structural refinement appear in Tables A1.1 - A1.6. In each case the data were processed $^{1}$ and corrected for Lorentz and polarization effects and absorption. Neutral atom scattering factors for all non-hydrogen atoms were taken from the International Tables for X-ray Crystallography. ${ }^{2,3}$ All structures were solved by direct methods ${ }^{4}$ and expanded using Fourier techniques. ${ }^{5}$ All non-hydrogen atoms were refined anisotropically except as noted in the Figure captions pertaining to each structure. Hydrogen atoms not refined were fixed in calculated positions with $\mathrm{C}-\mathrm{H}=0.98 \AA$. Dr. Brian O. Patrick solved structure 9, and the authors also acknowledge Dr. Christopher D. Carmichael for his assistance in solving the remaining structures. 
Table S-2. Crystallographic Data and Structure Refinement for $([\mathrm{NPN}] \mathrm{TaH})(\mu-\mathrm{H})_{2}\left(\mu-\eta^{1}: \eta^{2}-\right.$ $\left.\mathrm{NNSiH}_{2} \mathrm{Bu}\right)(\mathrm{Ta}[\mathrm{NPN}]) \quad(\mathbf{5}), \quad([\mathrm{NPN}] \mathrm{TaH})(\mu-\mathrm{N})\left(\mu-\mathrm{N}-\mathrm{SiH}_{2} \mathrm{Bu}\right)(\mathrm{Ta}[\mathrm{NPN}]) \quad(6), \quad$ and $([\mathrm{NPN}] \mathrm{Ta})_{2}\left(\mu-\mathrm{N}-\mathrm{SiH}_{2} \mathrm{Bu}\right)_{2}(4)$.

\begin{tabular}{|c|c|c|c|}
\hline & $\begin{array}{l}([\mathrm{NPN}] \mathrm{TaH})(\mu-\mathrm{H})_{2}\left(\mu-\eta^{1}: \eta^{2}-\right. \\
\left.\mathrm{NNSiH}_{2} \mathrm{Bu}\right)(\mathrm{Ta}[\mathrm{NPN}])(\mathbf{5})\end{array}$ & $\begin{array}{l}([\mathrm{NPN}] \mathrm{TaH})(\mu-\mathrm{N})(\mu-\mathrm{N}- \\
\left.\mathrm{SiH}_{2}{ }^{\mathrm{n}} \mathrm{Bu}\right) \mathrm{Ta}([\mathrm{NPN}])(\mathbf{6})\end{array}$ & $\begin{array}{l}([\mathrm{NPN}] \mathrm{Ta})_{2}(\mu-\mathrm{N}- \\
\left.\mathrm{SiH}_{2} \mathrm{Bu}\right)_{2}(\mathbf{4})\end{array}$ \\
\hline \multicolumn{4}{|l|}{ CCDC Registry } \\
\hline Formula & $\mathrm{C}_{52} \mathrm{H}_{76} \mathrm{~N}_{6} \mathrm{P}_{2} \mathrm{Si}_{5} \mathrm{Ta}_{2}$ & $\mathrm{C}_{52} \mathrm{H}_{74} \mathrm{~N}_{6} \mathrm{P}_{2} \mathrm{Si}_{5} \mathrm{Ta}_{2}$ & $\mathrm{C}_{56} \mathrm{H}_{84} \mathrm{~N}_{6} \mathrm{P}_{2} \mathrm{Si}_{6} \mathrm{Ta}_{2}$ \\
\hline $\begin{array}{l}\text { FW } \\
\text { Colour, habit } \\
\text { Crystal size, mm } \\
\text { Crystal system }\end{array}$ & $\begin{array}{l}1349.48 \\
\text { orange, prism } \\
0.25 \times 0.10 \times 0.05 \\
\text { orthorhombic }\end{array}$ & $\begin{array}{l}1347.48 \\
\text { yellow, needle } \\
0.25 \times 0.10 \times 0.05 \\
\text { triclinic }\end{array}$ & $\begin{array}{l}1433.67 \\
\text { red, block } \\
0.50 \times 0.40 \times 0.20 \\
\text { triclinic }\end{array}$ \\
\hline Space group & Pna21 (\#33) & $P_{\overline{1}(\# 2)}$ & $P_{\overline{1}(\# 2)}$ \\
\hline $\mathrm{a}, \AA$ & $27.600(6)$ & $11.804(5)$ & $11.843(1)$ \\
\hline $\mathrm{b}, \AA$ & $17.262(4)$ & $13.720(6)$ & $11.853(1)$ \\
\hline c, $\AA$ & $14.701(3)$ & $22.173(8)$ & $23.382(2)$ \\
\hline$\alpha, \operatorname{deg}$ & 90 & $95.92(3$ & $86.68(1)$ \\
\hline$\beta$, deg & 90 & $103.27(7)$ & $76.24(1)$ \\
\hline$\gamma, \operatorname{deg}$ & 90 & $11.61(4)$ & $79.36(1)$ \\
\hline $\mathrm{V}, \AA^{3}$ & $7004.0(3)$ & $3178.7(5)$ & $3132.8(5)$ \\
\hline Z & 4 & 2 & 2 \\
\hline$\rho_{\text {calc }}, \mathrm{g} / \mathrm{cm}^{3}$ & 1.28 & 1.504 & 1.52 \\
\hline $\mathrm{F}(000)$ & 2704.00 & 1448.00 & 1444.00 \\
\hline$\mu(\mathrm{MoK} \alpha), \mathrm{mm}^{-1}$ & 3.286 & 3.625 & 3.691 \\
\hline transmission factors & $0.6320-1.0000$ & $0.6125-1.0000$ & $0.6409-1.0000$ \\
\hline $2 \theta_{\max }, \operatorname{deg}$ & 55.84 & 50.10 & 59.6 \\
\hline total no. of reflns & 14507 & 16000 & 44484 \\
\hline no. of unique reflns & 11678 & 9745 & 20277 \\
\hline $\mathrm{R}_{\text {merge }}$ & 0.101 & 0.094 & 0.127 \\
\hline $\begin{array}{l}\text { no. reflns with } \mathrm{I} \geq \\
\text { no(I) }\end{array}$ & $10953(\mathrm{n}=2)$ & $6038(\mathrm{n}=2)$ & $17597(\mathrm{n}=2)$ \\
\hline no. of variables & 604 & 668 & 676 \\
\hline $\mathrm{R}\left(\mathrm{F}^{2}\right.$, all data $)$ & 0.0806 & 0.1119 & 0.0974 \\
\hline $\mathrm{R}_{\mathrm{w}}\left(\mathrm{F}^{2}\right.$, all data $)$ & 0.1462 & 0.1338 & 0.2114 \\
\hline $\mathrm{R}(\mathrm{F}, \mathrm{I}>\mathrm{n} \sigma(\mathrm{I}))$ & 0.0566 & 0.0575 & 0.0743 \\
\hline $\mathrm{R}_{\mathrm{w}}(\mathrm{F}, \mathrm{I}>\mathrm{n} \sigma(\mathrm{I}))$ & 0.1353 & 0.1193 & 0.1964 \\
\hline gof & 0.983 & 0.972 & 1.070 \\
\hline
\end{tabular}

Rigaku/ADSC CCD diffractometer, $\mathrm{R}=\Sigma|| \mathrm{F}_{\mathrm{o}}{ }^{2}|-| \mathrm{F}_{\mathrm{c}}{ }^{2}|| / \Sigma\left|\mathrm{F}_{\mathrm{o}}{ }^{2}\right| ; \mathrm{R}_{\mathrm{W}}=\left(\sum \mathrm{w}\left(\left|\mathrm{F}_{\mathrm{o}}{ }^{2}\right|-\left|\mathrm{F}_{\mathrm{c}}{ }^{2}\right|\right)^{2}\right.$ $\left./ \Sigma \mathrm{w}\left|\mathrm{F}_{\mathrm{o}}\right|^{2}\right)^{1 / 2}$.

For 6 , the data could not be indexed using the $\mathrm{d}^{*}$ TREK program, and the Twinsolve function in CrystalClear ${ }^{\mathrm{i}}$ determined the crystal was a two-component twin (components related by a rotation of $44^{\circ}$ normal to $(-0.1,-1.0$, -1.2 ) with the cited unit cell parameters. 
Table S-3. Crystallographic Data and Structure Refinement for $\left(\left[\mathrm{NP}\left(\mathrm{C}_{6} \mathrm{H}_{4}\right) \mathrm{N}\right] \mathrm{Ta}\right)(\mathrm{BuSiN}-\mu-$ $\mathrm{Si}(\mathrm{H}) \mathrm{Bu}-\mu-\mathrm{N})(\mathrm{Ta}[\mathrm{NPN}]), \quad(7), \quad([\mathrm{NPN}] \mathrm{TaH})(\mu-\mathrm{H})_{2}\left(\mu-\eta^{1}: \eta^{2}-\mathrm{NNSiH}_{2} \mathrm{Ph}\right)(\mathrm{Ta}[\mathrm{NPN}]), \quad 9$, and $[\mathrm{NPN}] \mathrm{Ta}\left(\mu-\mathrm{NSiH}_{2} \mathrm{Ph}\right)(\mu-\mathrm{NSiHPh}) \mathrm{Ta}[\mathrm{NPN}],(\mathbf{1 1})$.

\begin{tabular}{|c|c|c|c|}
\hline & $\begin{array}{l}\text { [NP(Ph)N]Ta(BuSiN- } \mu- \\
\mathrm{Si}(\mathrm{H}) \mathrm{Bu}-\mu-\mathrm{N}) \mathrm{Ta}[\mathrm{NPN}] \\
(7)\end{array}$ & $\begin{array}{l}{[\mathrm{NPN}] \mathrm{Ta}(\mathrm{H})(\mu-} \\
\mathrm{H})_{2}\left(\mu-\eta^{1}: \eta^{2}-\mathrm{N}_{2-}\right. \\
\left.\mathrm{SiH}_{2} \mathrm{Ph}\right) \mathrm{Ta}[\mathrm{NPN}](\mathbf{9})\end{array}$ & $\begin{array}{l}([\mathrm{NPN}] \mathrm{Ta})(\mu- \\
\left.\mathrm{NSiH}_{2} \mathrm{Ph}\right)(\mu- \\
\mathrm{NSiHPh})(\mathrm{Ta}[\mathrm{NPN}]),(\mathbf{1 1})\end{array}$ \\
\hline \multicolumn{4}{|l|}{ CCDC Registry } \\
\hline Formula & $\mathrm{C}_{56} \mathrm{H}_{82} \mathrm{~N}_{6} \mathrm{P}_{2} \mathrm{Si}_{6} \mathrm{Ta}_{2}$ & $\mathrm{C}_{57} \mathrm{H}_{79} \mathrm{~N}_{6} \mathrm{P}_{2} \mathrm{Si}_{5} \mathrm{Ta}_{2}$ & $\mathrm{C}_{60} \mathrm{H}_{74} \mathrm{~N}_{6} \mathrm{P}_{2} \mathrm{Si}_{6} \mathrm{Ta}_{2}$ \\
\hline FW & 1644.14 & 1369.47 & 1472.64 \\
\hline Colour, habit & yellow, irregular & orange, plate & Yellow, prism \\
\hline Crystal size, $\mathrm{mm}$ & $0.30 \times 0.20 \times 0.15$ & $0.20 \times 0.15 \times 0.05$ & $0.40 \times 0.25 \times 0.15$ \\
\hline Crystal system & triclinic & monoclinic & monoclinic \\
\hline Space group & $P 2_{1} / c$ & $P 2_{1} / \mathrm{n}(\# 14)$ & $P 2_{1} / \mathrm{c}(\# 14)$ \\
\hline $\mathrm{a}, \AA$ & $12.6466(7)$ & $14.825(6)$ & $12.885(5)$ \\
\hline $\mathrm{b}, \AA$ & $22.0411(12)$ & $29.26(3)$ & $22.800(3)$ \\
\hline $\mathrm{c}, \AA$ & $26.3579(19)$ & $15.831(8)$ & $21.860(6)$ \\
\hline$\alpha, \operatorname{deg}$ & 90.0 & 90.0 & 90 \\
\hline$\beta, \operatorname{deg}$ & $100.054(4)$ & $95.262(13)$ & $92.75(2)$ \\
\hline$\gamma, \operatorname{deg}$ & 90.0 & 90.0 & 90 \\
\hline $\mathrm{V}, \AA^{3}$ & $7234.31(53)$ & $6838(8)$ & $6414.7(14)$ \\
\hline $\mathrm{Z}$ & 4 & 5 & 4 \\
\hline$\rho_{\text {calc }}, \mathrm{g} / \mathrm{cm}^{3}$ & 1.51 & 1.663 & 1.525 \\
\hline $\mathrm{F}(000)$ & 3351.20 & 3420.00 & 2948.00 \\
\hline$\mu(\mathrm{MoK} \alpha), \mathrm{mm}^{-1}$ & 3.243 & 4.208 & 3.613 \\
\hline transmission factors & $0.5142-1.0000$ & $0.7112-1.0000$ & $0.6935-1.0000$ \\
\hline $2 \theta_{\max }, \mathrm{deg}$ & 55.76 & 55.76 & 55.8 \\
\hline total no. of reflns & 16189 & 14254 & 14435 \\
\hline no. of unique reflns & 16189 & 14254 & 14435 \\
\hline $\mathrm{R}_{\text {merge }}$ & 0.103 & 0.092 & 0.085 \\
\hline $\begin{array}{l}\text { no. reflns with } \mathrm{I} \geq \\
\text { no(I) }\end{array}$ & $11678(n=2)$ & $11834(\mathrm{n}=2)$ & $9484(\mathrm{n}=2)$ \\
\hline no. of variables & 712 & 642 & 697 \\
\hline $\mathrm{R}\left(\mathrm{F}^{2}\right.$, all data $)$ & 0.0972 & 0.0865 & 0.0803 \\
\hline $\mathrm{R}_{\mathrm{w}}\left(\mathrm{F}^{2}\right.$, all data $)$ & 0.2086 & 0.1682 & 0.0887 \\
\hline $\mathrm{R}(\mathrm{F}, \mathrm{I}>\mathrm{n} \sigma(\mathrm{I}))$ & 0.0726 & 0.0705 & 0.043 \\
\hline $\mathrm{R}_{\mathrm{w}}(\mathrm{F}, \mathrm{I}>\mathrm{n} \sigma(\mathrm{I}))$ & 0.1888 & 0.1552 & 0.0801 \\
\hline gof & 1.063 & 1.132 & 0.913 \\
\hline
\end{tabular}

Rigaku/ADSC CCD diffractometer, $\mathrm{R}=\Sigma|| \mathrm{F}_{\mathrm{o}}{ }^{2}|-| \mathrm{F}_{\mathrm{c}}{ }^{2}|| \Sigma\left|\mathrm{F}_{\mathrm{o}}{ }^{2}\right| ; \mathrm{R}_{\mathrm{W}}=\left(\Sigma \mathrm{W}\left(\left|\mathrm{F}_{\mathrm{o}}{ }^{2}\right|-\left|\mathrm{F}_{\mathrm{c}}{ }^{2}\right|\right)^{2}\right.$ $\left./ \Sigma \mathrm{W}\left|\mathrm{F}_{\mathrm{o}}\right|^{2}\right)^{1 / 2}$. 
Table S-4. Crystallographic Data and Structure Refinement for $([\mathrm{NPN}] \mathrm{Ta})_{2}\left(\mu-\mathrm{NSiH}_{2}{ }^{\mathrm{n}} \mathrm{Bu}\right)(\mu-$ $\left.\mathrm{NSiH}_{2} \mathrm{CH}_{2} \mathrm{CH}_{2} \mathrm{SiH}_{3}\right),(\mathbf{1 3}),\left(\left[\mathrm{NP}\left(\mathrm{C}_{6} \mathrm{H}_{4}\right) \mathrm{N}\right] \mathrm{Ta}\right)\left(\mathrm{N}-\mathrm{SiH}_{2} \mathrm{CH}_{2} \mathrm{CH}_{2} \mathrm{Si}(\mathrm{H})-\mu-\mathrm{N}\right)(\mathrm{Ta}[\mathrm{NPN}])(\mathbf{1 4})$, and $[\mathrm{NP}(\mathrm{Ph}) \mathrm{N}] \mathrm{Ta}\left(\mu-\mathrm{N}-\mathrm{SiH}_{2}{ }^{\mathrm{t}} \mathrm{Bu}\right)\left({ }^{\mathrm{t}} \mathrm{BuSi}(\mathrm{H}) \mathrm{N}=\right) \mathrm{Ta}[\mathrm{NPN}]$.

\begin{tabular}{|c|c|c|c|}
\hline & $\begin{array}{l}([\mathrm{NPN}] \mathrm{Ta})_{2}\left(\mu-\mathrm{NSiH}_{2}{ }^{\mathrm{n}} \mathrm{Bu}\right)(\mu- \\
\left.\mathrm{N} \mathrm{SiH}_{2} \mathrm{CH}_{2} \mathrm{CH}_{2} \mathrm{SiH}_{3}\right) \\
(\mathbf{1 3}) \\
\end{array}$ & $\begin{array}{l}\mathrm{NP}(\mathrm{Ph}) \mathrm{N}] \mathrm{Ta}(\mathrm{N}- \\
\mathrm{SiH}_{2} \mathrm{CH}_{2} \mathrm{CH}_{2} \mathrm{Si}(\mathrm{H})-\mu- \\
\mathrm{N}) \mathrm{Ta}[\mathrm{NPN}](\mathbf{1 4}) \\
\end{array}$ & $\begin{array}{l}{[\mathrm{NP}(\mathrm{Ph}) \mathrm{N}] \mathrm{Ta}(\mu-\mathrm{N}-} \\
\left.\mathrm{SiH}_{2}{ }^{\mathrm{t}} \mathrm{Bu}\right)\left({ }^{\mathrm{t}} \mathrm{BuSi}(\mathrm{H}) \mathrm{N}=\right) \mathrm{Ta} \\
{[\mathrm{NPN}]}\end{array}$ \\
\hline \multicolumn{4}{|l|}{ CCDC Registry } \\
\hline Formula & $\mathrm{C}_{53} \mathrm{H}_{78} \mathrm{~N}_{6} \mathrm{Ta}_{2} \mathrm{P}_{2} \mathrm{Si}_{6}$ & $\mathrm{C}_{56} \mathrm{H}_{70} \mathrm{~N}_{6} \mathrm{Ta}_{2} \mathrm{P}_{2} \mathrm{Si}_{6}$ & $\mathrm{C}_{56} \mathrm{H}_{84} \mathrm{~N}_{6} \mathrm{Ta}_{2} \mathrm{P}_{2} \mathrm{Si}_{6}$ \\
\hline $\begin{array}{l}\text { FW } \\
\text { Colour, habit } \\
\text { Crystal size, } \mathrm{mm} \\
\text { Crystal system }\end{array}$ & $\begin{array}{l}1391.60 \\
\text { red, plate } \\
0.40 \times 0.40 \times 0.05 \\
\text { triclinic }\end{array}$ & $\begin{array}{l}1347.52 \\
\text { yellow, prism } \\
0.25 \times 0.20 \times 0.15 \\
\text { monoclinic }\end{array}$ & $\begin{array}{l}1347.54 \\
\text { pink, prism } \\
0.35 \times 0.30 \times 0.25 \\
\text { monoclinic }\end{array}$ \\
\hline Space group & $P_{\overline{1}(\# 2)}$ & $P 2_{1} / \mathrm{a}(\# 14)$ & $P 2_{1} / \mathrm{c}(\# 14)$ \\
\hline $\mathrm{a}, \AA$ & $11.8417(4)$ & $20.6518(30)$ & $12.7146(54)$ \\
\hline $\mathrm{b}, \AA$ & $11.9131(3)$ & $12.4903(17)$ & $21.4708(48)$ \\
\hline $\mathrm{c}, \AA$ & $23.1431(11)$ & $22.0439(33)$ & $24.0704(51)$ \\
\hline$\alpha, \operatorname{deg}$ & $77.561(7)$ & 90.0 & 90 \\
\hline$\beta, \operatorname{deg}$ & $86.578(8)$ & $100.148(4)$ & $104.354(19)$ \\
\hline$\gamma, \operatorname{deg}$ & $78.877(7)$ & 90.0 & 90 \\
\hline $\mathrm{V}, \AA^{3}$ & $3127.74(7)$ & $5597.21(43)$ & $6365.91(325)$ \\
\hline Z & 2 & 4 & 4 \\
\hline$\rho_{\text {calc }}, \mathrm{g} / \mathrm{cm}^{3}$ & 1.48 & 1.60 & 1.41 \\
\hline $\mathrm{F}(000)$ & 1395.60 & 2687.30 & 2695.30 \\
\hline$\mu(\operatorname{MoK} \alpha), \mathrm{mm}^{-1}$ & 3.700 & 4.132 & 3.615 \\
\hline transmission factors & $0.7921-1.0000$ & $0.6386-1.0000$ & $0.6748-1.0000$ \\
\hline $2 \theta_{\max }, \operatorname{deg}$ & 55.8 & 55.8 & 55.8 \\
\hline total no. of reflns & 12673 & 12469 & 13426 \\
\hline no. of unique reflns & 12673 & 12469 & 13426 \\
\hline$R_{\text {merge }}$ & 0.000 & 0.000 & 0.000 \\
\hline $\begin{array}{l}\text { no. reflns with } \mathrm{I} \geq \\
\text { no(I) }\end{array}$ & $10216(n=2)$ & $9193(n=2)$ & $10345(\mathrm{n}=2)$ \\
\hline no. of variables & 625 & 595 & 649 \\
\hline $\mathrm{R}\left(\mathrm{F}^{2}\right.$, all data $)$ & 0.079 & 0.107 & 0.059 \\
\hline $\mathrm{R}_{\mathrm{w}}\left(\mathrm{F}^{2}\right.$, all data $)$ & 0.0176 & 0.222 & 0.098 \\
\hline $\mathrm{R}(\mathrm{F}, \mathrm{I}>\mathrm{n} \sigma(\mathrm{I}))$ & 0.065 & 0.085 & 0.037 \\
\hline $\mathrm{R}_{\mathrm{w}}(\mathrm{F}, \mathrm{I}>\mathrm{n} \sigma(\mathrm{I}))$ & 0.156 & 0.205 & 0.092 \\
\hline gof & 1.106 & 1.084 & 1.033 \\
\hline
\end{tabular}

Rigaku/ADSC CCD diffractometer, $\mathrm{R}=\Sigma|| \mathrm{F}_{\mathrm{o}}{ }^{2}|-| \mathrm{F}_{\mathrm{c}}{ }^{2}|/ \Sigma| \mathrm{F}_{\mathrm{o}}{ }^{2} \mid ; \mathrm{R}_{\mathrm{W}}=\left(\Sigma \mathrm{w}\left(\left|\mathrm{F}_{\mathrm{o}}{ }^{2}\right|-\left|\mathrm{F}_{\mathrm{c}}{ }^{2}\right|\right)^{2}\right.$ $\left./ \Sigma \mathrm{w}\left|\mathrm{F}_{\mathrm{o}}{ }^{2}\right|^{2}\right)^{1 / 2}$. 


\section{References}

1) teXsan Crystal Structure Analysis Package;; Molecular Structure Corp.: The Woodlands, TX, 1995.

2) International Tables for X-Ray Crystallography; Kluwer Academic: Boston, MA, 1992; Vol. C, pp 200-206.

3) International Tables for X-Ray Crystallography; Kynoch Press: Birmingham, U. K. (present distributer Kluwer Academic: Boston, MA), 1974; Vol. IV, pp 99-102.

4) Altomare, A.; Burla, M. C.; Cammali, G.; Cascarano, M.; Giacovazzo, C.; Guagliardi, A.; Moliterni, A. G. G.; Polidori, G.; Spagna, A. SIR97: a new tool for crystal structure determination and refinement, 1999. 1999.

5) Beurskens, P. T.; Admiraal, G.; Beurskens, G.; Bosman, W. P.; de Gelder, R.; Israel, R.; Smits, J. M. M. DIRDIF94; The DIRDIF-94 program system, Technical Report of the Crystallography Laboratory; University of Nijmegen: The Netherlands, 1994.

6) CrystalClear: Version 1.3.5b20. Molecular Structure Corporation (2002). 